\title{
The use of peak over threshold methods to characterise blood glucose curves
}

\author{
Mátyás Szigeti \\ Physiological Controls Researc \\ Óbuda University \\ Budapest, Hungary \\ szigeti.matyas@phd.uni-obuda.hu \\ Tamás Ferenci \\ Physiological Controls Research Center \\ Óbuda University \\ Budapest, Hungary \\ ferenci.tamas@nik.uni-obuda.hu \\ Levente Kovács \\ Physiological Controls Research Center \\ Óbuda University \\ Budapest, Hungary \\ kovacs.levente@nik.uni-obuda.hu
}

\begin{abstract}
In contrast to regular statistics which focuses on the typical part of the data and use metrics to describe that part (usually the mean or variance), there is a branch of statistics which focuses on the extreme and thus rare events. The use of extreme value statistics allows us to fit models on this part of the data and like ,regular" statistics, enables us to calculate estimates and predictions, but in this case for extreme values. These methods are frequently used in fields like meteorology and finance where the extreme events has large impact despite their rarity. Because of this rarity, however, only a small fraction of the data can be used so much higher sample size is required for such analysis - thus fields with a large amount of historical data have an advantage.
\end{abstract}

This factor limited the use of extreme value statistics in biomedical field where available technology and costs are strong limitations at measuring most of the biomarkers until recently.

Blood glucose level is one of the exceptions nowadays, as with recent advancements it can be monitored for relatively long time and with high frequency for a patient. Additionally, extreme values of blood glucose levels (both high and low) are associated with - chronic or acute - complications of diabetes.

This paper aims to demonstrate that the use of extreme value statistics could be a possible way to characterize blood glucose curves. In addition to providing a metric for the state of the patient, it allows the comparison of the performance of artificial pancreas models.

Peak over threshold method was used to model extreme values of a simulated dataset containing 1440 measurements of 99 patients with $250 \mathrm{mg} / \mathrm{dl}$ as threshold. Probabilities for exceeding the clinically relevant levels of $270 \mathrm{mg} / \mathrm{dl}$ (cognitive symptoms expected) and $600 \mathrm{mg} / \mathrm{dl}$ (diabetic hyperosmolar syndrome) were calculated and were $23.9 \%$ and $8.0 \cdot 10^{-6} \%$ respectively in the region above the threshold $(250 \mathrm{mg} / \mathrm{dl})$. Through these estimates it is possible to compare different controllers.

Index Terms - extreme value statistics, artificial pancreas, diabetes, generalised Pareto distribution, peak over threshold, cgms

\section{INTRODUCTION}

In statistics the general aim is to draw conclusions about the population through analysing a sample taken from that population. We could describe a population by giving all of

Project no. 2019-1.3.1-KK-2019-00007. has been implemented with the support provided from the National Research, Development and Innovation Fund of Hungary, financed under the 2019-1.3.1-KK funding scheme. This project has received funding from the European Research Council (ERC) under the European Union's Horizon 2020 Research and Innovation Programme (Grant Agreement no. 679681). its elements but in almost all cases that would be to much data, or, even more typically, the population is infinite, given by its distribution. Usually we focus our interest to the most relevant part of the population which are often the most common elements. Mode exactly does that by definition, the mean and the median is often close to it for the most common distributions and where they are not, we tend to use other statistics like geometric mean instead of them. Mean could be biased by the outliers but in these cases we try to deal with them often by simply dropping the outliers but we never try to get rid of the values from the center despite that we aware that the outliers could hold very valuable information. However, their rarity makes them less reliable.

In medical statistics, outliers have an even a more important role as in some sense diseases are always some non-normal conditions leading to some biomarkers reach very high or very low levels. Of course if our sample contains ill people only these values will not be extreme for our sample but common extremes of some biomarkers could be very meaningful in terms of the outcomes of the disease.

There is a branch of statistics which focuses on the extreme and - by that nature - rare events. This is called Extreme Value Statistics (EVS) or Extreme Value Theory (EVT). This originates in the works of Ronald Fisher, Leonard Henry Caleb Tippett and Boris Vladimirovich Gnedenko, the central result of which is summarized in the so-called Fisher-Tippett-Gnedenko theorem [1], [2]. Because these events or observations are rare, and form just a fraction of our total sample, their analysis is exceptionally difficult.

The EVS is often used in architecture, weather and climate analysis and finance but rarely in medical statistics. One important reason for this is the lack of data on extremes and the cost of obtaining them compared to natural factors like weather which is simple to observe and abundant data are (publicly) available, with daily or even higher frequency for very remote places of Earth and from decades ago. Even for very serious conditions, important biomarkers are usually those that require some sample taken that might be measured for patient maybe a dozen times.

Electronic patient monitoring and electronic health records increase the amount of data in medical research as well. multiples by each year [3]. Some areas benefit from this more 
than the others.

Measuring or monitoring blood sugar (glucose) levels became available and more common relatively long time ago [4]. With the introduction of continuous glucose monitoring system (CGMS), even high-frequency (typically 5 minutes sampling time), longer-term (up to a week) measurement became available [5].

Diabetic patients lost the ability to maintain normal glucose levels. Glucose levels that are not extreme to cause acute problems, but are higher than normal can still results in very severe effects including blindness or the loss of limbs on the long term. Extremely high or low levels of glucose can lead to acute medical emergencies, including the loss of consciousness, onset of coma or even death [6]. Artificial control is difficult to achieve as reactions to treatment or any other factors (activity, caloric intake) are different from person to person. Real-time control of glucose level is the aim of the Artificial Pancreas (AP) [7]. These machines and algorithms are able to control blood glucose levels by injecting insulin to the blood stream and measuring the results as the same time. The proper control is still an issue and there are a lot of different algorithms out there.

The current practice of summarizing continuously measured blood glucose curves involves presenting simple indicators, mostly focusing on variability itself, irrespectively of how extreme the blood glucose level was.

With the use of EVT we should be able to give an estimate for the probability of blood glucose level exceeding a certain threshold and also give confidence intervals (CI) like we do in regular statistics. This might catch the essence of the problem better as this would describe the chance of an adverse event such as losing consciousness thus making the comparison of controllers more relevant.

Our aim is to demonstrate that EVT enables us to characterize a blood glucose curve, which can be used - among others - to create patient-level summary metrics and to assess the performance of artificial pancreas algorithms in a more precise and practical way.

\section{Methods}

\section{A. Extreme Value Theorem}

There are two main approaches when it comes to the analysis of extreme values and both approaches are relevant in glucose level assessment.

The block maxima (BM) approach splits the data to equal sized blocks and takes the maximum of each block as a secondary sample to analyse. The Peak Over Threshold (POT) approach, that will be applied in the present paper, uses a cutoff level and takes all the values over that level as a secondary sample. Generalised Pareto distribution was first introduced by James Pickands III in 1975 [8] who has shown that it describes the behavior of the extreme values, formally:

$$
P(X-u<y \mid X>u) \approx 1-\left(1+\frac{\xi y}{\tilde{\sigma}}\right)^{-1 / \xi},
$$

where $\xi$ is the shape and $\sigma$ is the scale parameter.

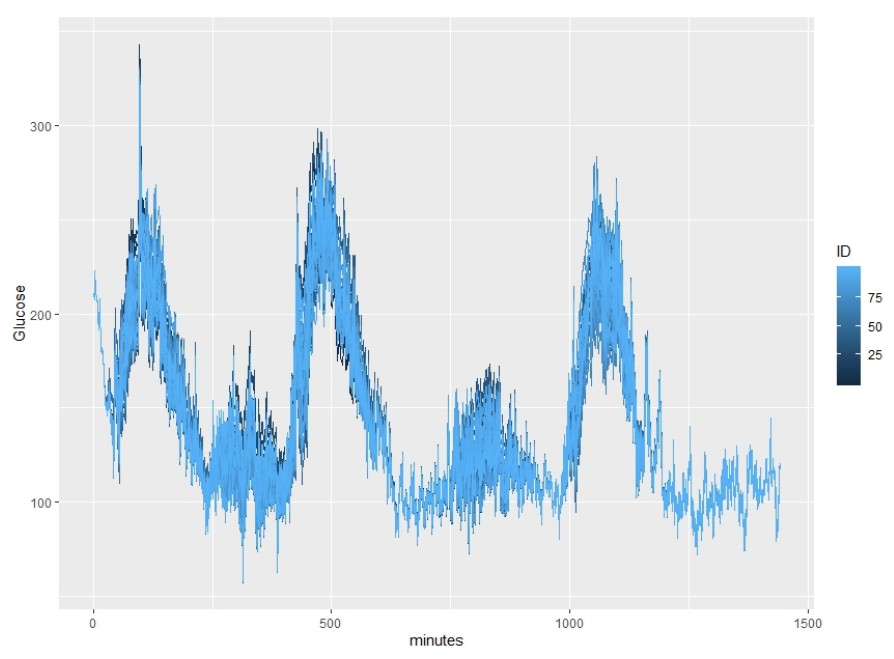

Fig. 1. Plot of 1440 simulated blood glucose measurements of 99 patients

\section{B. Data and Programs used}

The sample was taken using simulation from the UVA/PADOVA Type 1 diabetes simulator [9]. This contains 1440 measurements of 99 simulated patients. Assuming 1 minute between the measurements, this covers a whole day for each simulated patient.

The analysis was conducted using the $\mathrm{R}$ statistical program package version 3.6.2 [10] and ExtRemes 2.0 package [11].

\section{Results}

The simulated measurements of the 99 patients are shown in Figure 1. The median glucose level across the whole dataset was $123 \mathrm{mg} / \mathrm{dl}$ with a range of 57-343 mg/dl.

First a (sampling) threshold level was chosen using a mean excess plot (Figure 2) and the threshold range plot (Figure 3). This is a key element of the analysis because if the threshold is too high then we lose too much data and the remaining while characteristic for the extremes - might not be sufficient for the analysis, on the other hand, if the threshold is too low then the model will be fitted to the non-extreme parts of the data. The mean excess plot on Fig. 2 shows an ideal (flat) region at $250 \mathrm{mg} / \mathrm{dl}$.

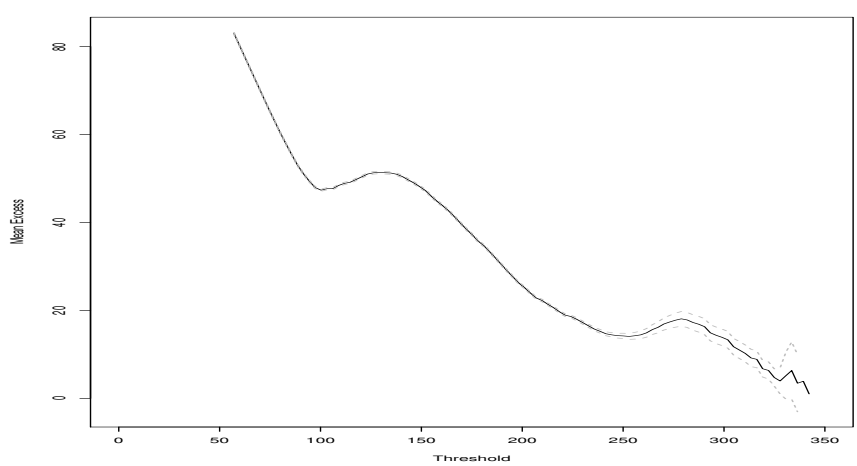

Fig. 2. Mean excess plot show that $250 \mathrm{mg} / \mathrm{dl}$ was an ideal threshold level 

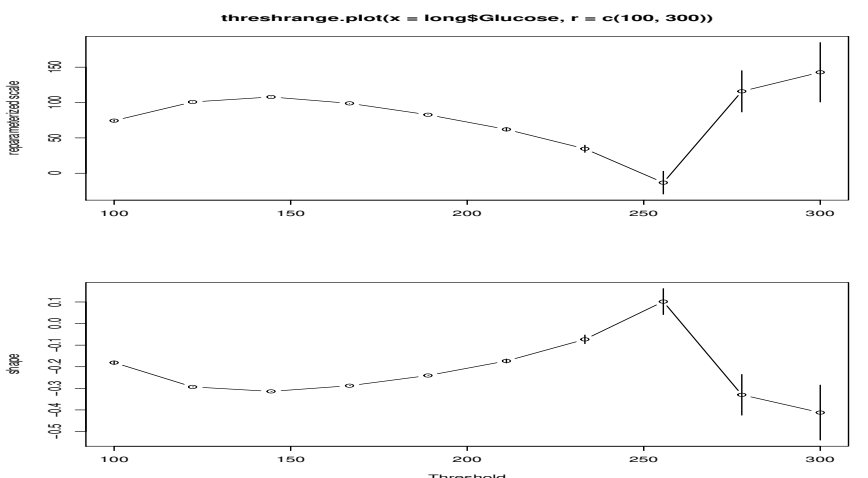

Fig. 3. Thresh range plot shows the scale and shape parameter values and their $95 \%$ CI for different thresholds

A POT model was fitted to the simulated dataset. The goodness of fit was assessed graphically by various diagnostic plots shown on Fig. 4. The model fitted well to a certain level around $320 \mathrm{mg} / \mathrm{dl}$ and it seems above that level our data is less extreme than what GP distribution would suggest. This was expected because of the nature of the simulated data additionally there were just a very few measurements above $300 \mathrm{mg} / \mathrm{dl}$. For this reason $270 \mathrm{mg} / \mathrm{dl}$ was chosen as an investigated threshold which is within the available data's range and symptoms as cognitive impairment are expected at this level [12]. We chose $600 \mathrm{mg} / \mathrm{dl}$ as a secondary limit which is way beyond this range, but is an important physiological limit for diabetic hyperosmolar syndrome which leads to coma for $25-50 \%$ of the patients [13].

The fitted model assumes a Generalised Pareto distribution for the values above the threshold with 13.46 (95\% CI: 12.66 , $14.27)$ as scale and 0.0530 (95\% CI: $0.0088,0.0973)$ as shape parameters. According to the fitted model there is $23.9 \%$ probability to exceed $270 \mathrm{mg} / \mathrm{dl}$ and $8.0 \cdot 10^{-6 \%}$ probability to exceed $600 \mathrm{mg} / \mathrm{dl}$ for a new peak above $250 \mathrm{mg} / \mathrm{dl}$.

\section{Discussion}

Several metrics are used in clinical practice to describe the variability of a patient's blood glucose levels. These include the Mean Amplitude of Glycaemic Excursions (MAGE) [14], the Continuous Net Overall Glycaemic Action (CONGA) [15], simple coefficient of variation or interquartile range of blood glucose levels, or percentage time spent above or below a certain threshold [16].

These metrics, however, mostly focus on overall variability, not specifically on extremities. (I.e., a patient's variability can be very high, even if the blood glucose level is never in the extreme range, or conversely, the patient can be in extreme range with very low variability.) Thus, these metrics are not appropriate to capture this aspect. Those that do capture this aspect are usually very simple indicators (like percentage time spent in extreme range, or the CVGA plot [17] which is essentially the same, but in graphical form), in contrast to which our approach is based on a much more sophisticated statistical foundation.
Previously in the biomedical field EVT was rarely used. Although EVT was used to analyse cholesterol levels [18], pneumonia and influenza deaths [19], the lack off sufficient data is a serious limitation for the EVT in biomedical science.

\section{CONCLUSION}

In this paper the concept of using EVT to assess the quality of glucose control based on a CGMS curve been successfully proven. However there are many important points are yet to be answered.

POT analysis was used which only reflects values above a certain threshold and within that tail of the data it enables to calculate estimates for the probability of reaching certain extremes. This is useful to compare e.g. different AP controllers comprehensively, but does not take into account the extremes occurrence in time, therefore it is less suitable for patient-level characterization of glycaemia.

The other main approach of EVT, the block maxima approach focuses on the distributions of maxima in time so it might be more relevant clinically to give a metric for each patient but the application is yet to explore.

One of the main limitation was that the threshold of diabetic hyperosmolar syndrome was very far from the upper boundaries of the data thus the results might be unreliable for the higher threshold.

Additionally we did not take into account the dependencies of the measurements. [20] Neighbouring or close measurements within each patient strongly correlated because of physiological reasons and that was taken into account in the simulation but not in our analysis. A further development could be to use methods which are able to account for these correlations in the estimates. Additionally, too low blood glucose levels can also lead to life-threatening conditions thus a natural aspect to extend our investigations to assess not just the peaks but the extremely low measurements.

By the nature of the simulated data there were unrealistic similarities between the patients. Their starting value was the same and without any glucose or insulin input they reached the same reference level (middle and last section of Fig. 1). The periodicity of glucose inputs ("meals") were the same for all patients. It will be challenging to obtain significant amount of real-life data but it will be the most important step forward to try these methods on real-life data. We expect that in this case the model fitting will be better on non-simulated data. 

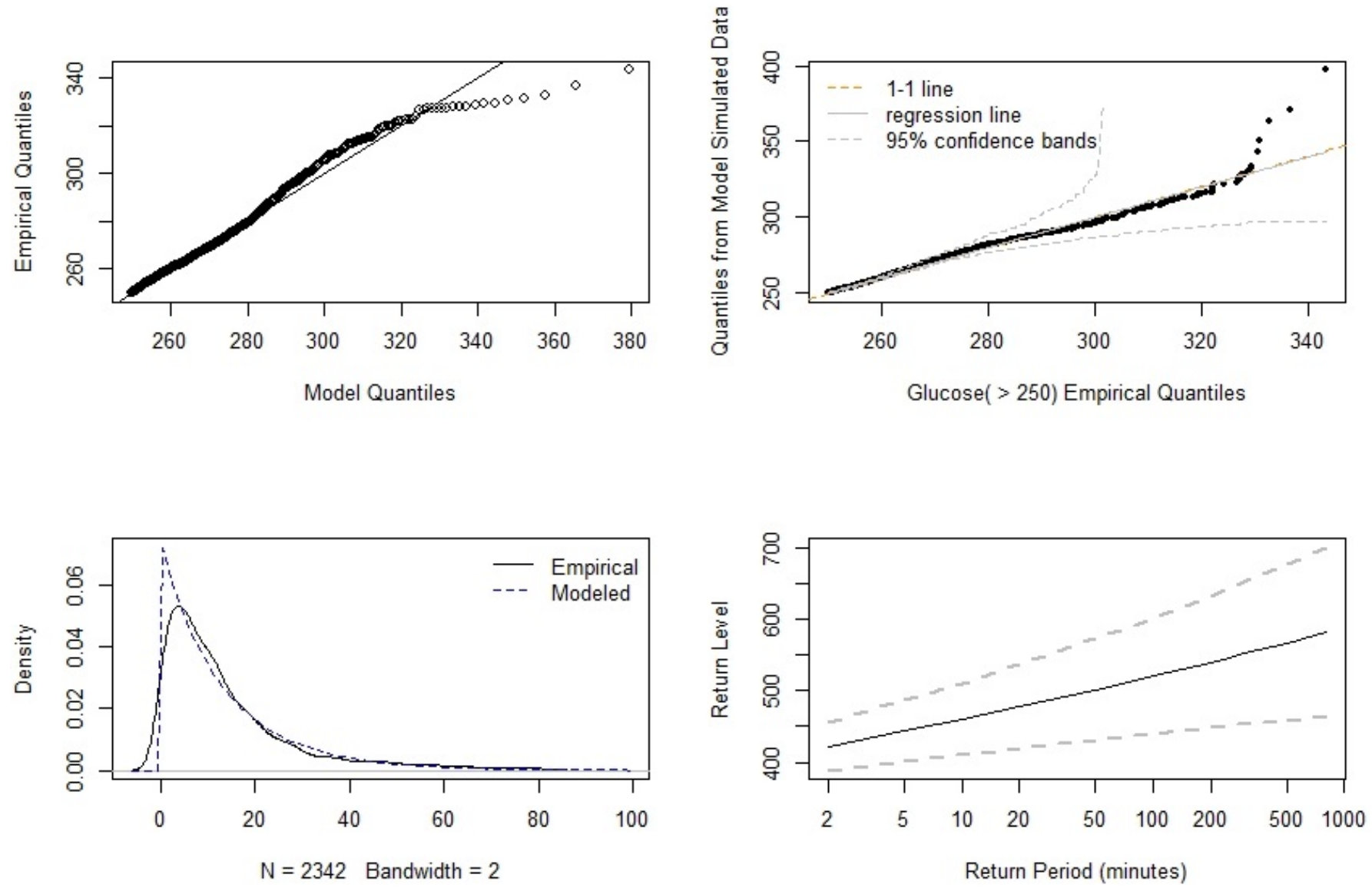

Fig. 4. Model diagnostics plots

\section{REFERENCES}

[1] R. A. Fisher and L. H. C. Tippett, "Limiting forms of the frequency distribution of the largest or smallest member of a sample," Mathematical Proceedings of the Cambridge Philosophical Society, vol. 24, no. 2, pp. 180-190, Apr. 1928. [Online]. Available: https://www.cambridge.org/core/journals/mathematicalproceedings-of-the-cambridge-philosophical-society/article/limitingforms-of-the-frequency-distribution-of-the-largest-or-smallest-memberof-a-sample/7BE8DE65FCDFC3ABECFE1054DFB56CB5

[2] B. Gnedenko, "Sur la distribution limite du terme maximum d'une serie aleatoire," Annals of Mathematics, vol. 44, no. 3, pp. 423-453, 1943. [Online]. Available: http://www.jstor.org/stable/1968974

[3] J. K. Wang, D. Ouyang, J. Hom, J. Chi, and J. H. Chen, "Characterizing electronic health record usage patterns of inpatient medicine residents using event log data,' PLOS ONE, vol. 14, no. 2, pp. 1-7, 022019. [Online]. Available: https://doi.org/10.1371/journal.pone.0205379

[4] S. Clarke and J. Foster, "A history of blood glucose meters and their role in self-monitoring of diabetes mellitus," British journal of biomedical science, vol. 69, no. 2, pp. 83-93, 2012.

[5] B. W. Bode, "Clinical utility of the continuous glucose monitoring system," Diabetes Technology \& Therapeutics, vol. 2, no. 1, Supplement 1, pp. 35-41, 2000.

[6] J. Wang, D. E. Williams, K. V. Narayan, and L. S. Geiss, "Declining death rates from hyperglycemic crisis among adults with diabetes, us, 1985-2002," Diabetes care, vol. 29, no. 9, pp. 2018-2022, 2006.

[7] C. Cobelli, E. Renard, and B. Kovatchev, "Artificial pancreas: past, present, future," Diabetes, vol. 60, no. 11, pp. 2672-2682, 2011.

[8] J. Pickands III et al., "Statistical inference using extreme order statistics," the Annals of Statistics, vol. 3, no. 1, pp. 119-131, 1975.
[9] C. D. Man, F. Micheletto, D. Lv, M. Breton, B. Kovatchev, and C. Cobelli, "The uva/padova type 1 diabetes simulator: new features," Journal of diabetes science and technology, vol. 8, no. 1, pp. 26-34, 2014.

[10] R Core Team, R: A Language and Environment for Statistical Computing, R Foundation for Statistical Computing, Vienna, Austria, 2019. [Online]. Available: https://www.R-project.org/

[11] E. Gilleland and R. W. Katz, "extRemes 2.0: An extreme value analysis package in R,” Journal of Statistical Software, vol. 72, no. 8, pp. 1-39, 2016.

[12] I. Pais, M. Hallschmid, K. Jauch-Chara, S. M. Schmid, K. M. Oltmanns, A. Peters, J. Born, and B. Schultes, "Mood and Cognitive Functions During Acute Euglycaemia and Mild Hyperglycaemia in Type 2 Diabetic Patients," Experimental and Clinical Endocrinology \& Diabetes, vol. 115, no. 01, pp. 42-46, Jan. 2007. [Online]. Available: http://www.thieme-connect.de/DOI/DOI?10.1055/s-2007-957348

[13] F. J. Pasquel and G. E. Umpierrez, "Hyperosmolar Hyperglycemic State: A Historic Review of the Clinical Presentation, Diagnosis, and Treatment," Diabetes Care, vol. 37, no. 11, pp. 3124-3131, Nov. 2014. [Online]. Available: https://www.ncbi.nlm.nih.gov/pmc/articles/PMC4207202/

[14] F. J. Service, G. D. Molnar, J. W. Rosevear, E. Ackerman, L. C. Gatewood, and W. F. Taylor, "Mean amplitude of glycemic excursions, a measure of diabetic instability," Diabetes, vol. 19, no. 9, pp. 644-655, 1970. [Online]. Available: https://diabetes.diabetesjournals.org/content/19/9/644

[15] C. McDonnell, S. Donath, S. Vidmar, G. Werther, and F. Cameron, "A novel approach to continuous glucose analysis utilizing glycemic variation," Diabetes Technology \& Therapeutics, vol. 7, no. 2, pp. 253-263, 2005, pMID: 15857227. [Online]. Available: https://doi.org/10.1089/dia.2005.7.253 
[16] G. Marics, Z. Lendvai, C. Lódi, L. Koncz, D. Zakariás, G. Schuster, B. Mikos, C. Hermann, A. J. Szabó, and P. Tóth-Heyn, "Evaluation of an open access software for calculating glucose variability parameters of a continuous glucose monitoring system applied at pediatric intensive care unit," Biomedical engineering online, vol. 14, no. 1, p. 37, 2015.

[17] L. Magni, D. M. Raimondo, C. D. Man, M. Breton, S. Patek, G. De Nicolao, C. Cobelli, and B. P. Kovatchev, "Evaluating the efficacy of closed-loop glucose regulation via control-variability grid analysis," Journal of diabetes science and technology, vol. 2, no. 4, pp. 630-635, 2008.

[18] P. Bermudez and Z. Mendes, "Extreme value theory in medical sciences: Modeling total high cholesterol levels," Journal of statistical theory and practice, vol. 6, pp. 468-491, 092012.

[19] M. Thomas, M. Lemaitre, M. L. Wilson, C. Viboud, Y. Yordanov, H. Wackernagel, and F. Carrat, "Applications of extreme value theory in public health," PloS one, vol. 11, no. 7, 2016.

[20] T. Ferenci, J. Sápi, and L. Kovács, "Modelling tumor growth under angiogenesis inhibition with mixed-effects models," Acta Polytechnica Hungarica, vol. 14, no. 1, pp. 221-234, jan 2017. 
M. Szigeti et al. - The use of peak over threshold methods to characterise blood glucose curves 